University of Nebraska - Lincoln

DigitalCommons@University of Nebraska - Lincoln

Changes in mineral concentrations and phosphorus profile during dry-grind processing of corn into ethanol

KeShun Liu

USDA-ARS

Jianchun Han

Northeast Agricultural University

Follow this and additional works at: https://digitalcommons.unl.edu/usdaarsfacpub

Part of the Agricultural Science Commons

Liu, KeShun and Han, Jianchun, "Changes in mineral concentrations and phosphorus profile during drygrind processing of corn into ethanol" (2011). Publications from USDA-ARS / UNL Faculty. 862.

https://digitalcommons.unl.edu/usdaarsfacpub/862

This Article is brought to you for free and open access by the U.S. Department of Agriculture: Agricultural Research Service, Lincoln, Nebraska at DigitalCommons@University of Nebraska - Lincoln. It has been accepted for inclusion in Publications from USDA-ARS / UNL Faculty by an authorized administrator of DigitalCommons@University of Nebraska - Lincoln. 


\title{
Changes in mineral concentrations and phosphorus profile during dry-grind processing of corn into ethanol
}

\author{
KeShun Liu ${ }^{\mathrm{a}, *}$, Jianchun Han ${ }^{\mathrm{b}}$ \\ ${ }^{a}$ Grain Chemistry and Utilization Laboratory, National Small Grains and Potato Germplasm Research Unit, USDA-ARS, 1691 S. 2700 West, Aberdeen, ID 83210, USA \\ ${ }^{\mathrm{b}}$ College of Food Science, Northeast Agricultural University, 59 Mucai St. Xiangfang District, Harbin, Heilongjian 150030, China
}

\section{A R T I C L E I N F O}

\section{Article history:}

Received 16 July 2010

Received in revised form 12 October 2010

Accepted 14 October 2010

Available online 20 October 2010

\section{Keywords:}

DDGS

Dry grind

Minerals

Phytate

Fuel ethanol

\begin{abstract}
A B S T R A C T
For determining variation in mineral composition and phosphorus (P) profile among streams of dry-grind ethanol production, samples of ground corn, intermediate streams, and distillers dried grains with solubles (DDGS) were obtained from three commercial plants. Most attributes (dry matter concentrations) increased significantly from corn to cooked slurry but fermentation caused most significant increase in all attributes. During centrifugation, more minerals went into thin stillage than wet grains, making minerals most concentrated in the former. Mineral increase in DDGS over corn was about 3 fold, except for $\mathrm{Na}, \mathrm{S}, \mathrm{Ca}$, and Fe. The first three had much higher fold of increase, presumably due to exogenous addition. During fermentation, phytate P and inorganic P had 2.54 and 10.37 fold of increase over corn, respectively, while relative to total P, \% phytate P decreased and \% inorganic P increased significantly. These observations suggest that phytate underwent some degradation, presumably due to activity of yeast phytase.
\end{abstract}

Published by Elsevier Ltd.

\section{Introduction}

Increasing demand for ethanol as a fuel additive and decreasing dependency on fossil fuels have resulted in a dramatic increase in the amount of starchy grains used for ethanol production. A major process for making fuel ethanol from grains (mainly corn) is the dry-grind method, in which all kernel components are processed through several sequential steps, including grinding, cooking, liquefaction, saccharification, fermentation, distillation, and coproduct recovery (Bothast and Schlicher, 2005). Distillers dried grains with solubles (DDGS) are the major co-product available for marketing. Production of DDGS has increased significantly in recent years, as the number of dry-grind ethanol production facilities increased.

High concentrations and high variation of minerals in DDGS impact its value and end use as animal feed (Spiehs et al., 2002; Batal and Dale, 2003; Belyea et al., 2006). High concentration can lead to not only nutritional disorders (Niles et al., 2002) but also excessive mineral concentrations in wastes (Spiehs and Varel, 2009), while high variation in mineral contents makes accurate diet formulation difficult because assumed concentrations could be different from actual concentrations. For examples, high sulfur (S) content in diets has been associated with thiamine deficiency, which in turn causes polioencephalomalacia (PEM) in ruminants (Gould, 1998; Niles

\footnotetext{
* Corresponding author. Tel.: +1 208397 4162; fax: +1 2083974165

E-mail address: Keshun.Liu@ars.usda.gov (K.S. Liu).
}

et al., 2002). It has also been linked, together with high nitrogen $(\mathrm{N})$, to increased odorant production in manure (Spiehs and Varel, 2009). High phosphorus (P) concentration in DDGS, which ranges from $0.5 \%$ to $1.0 \%$ (Spiehs et al., 2002), has been shown to cause increased $\mathrm{P}$ excretion in livestock wastes, which in turn increases the amount of land necessary to utilize manure P (Koelsch and Lesoing, 1999; Spiehs and Varel, 2009). Therefore, high DDGS inclusion in rations for certain animals has been avoided because of potential problems with PEM and/or environmental concerns.

Bioavailability of $\mathrm{P}$ in animal feed is another important factor that affects retention of $P$ in ingested feeds by non ruminant animals and the amount of $P$ excreted in wastes. P bioavailability is determined by its chemical forms. Grains and their byproducts contain different forms of $\mathrm{P}$, including inorganic $\mathrm{P}$, phytate $\mathrm{P}$, and the rest of $P$ (i.e. remaining $P$ ). Phytate or inositol hexakisphosphate is found in most cereal seeds. It is the main storage form of $P$ in grains (Lott et al., 2000). Inorganic P (also known as phosphate $\mathrm{P}$ or free $\mathrm{P}$ ) has higher bioavailability than phytate $\mathrm{P}$. The rest of $P$ represents the sum of all $P$-containing compounds in a sample other than phytate $\mathrm{P}$ and inorganic $\mathrm{P}$. It includes, for example, $\mathrm{P}$ found in DNA, RNA, proteins, phospholipids and starch. Since phytate $\mathrm{P}$ is not well utilized by monogastric animals, it contributes to increased P discharge into the environment (He et al., 2009). In addition, phytate has been shown to interact directly and indirectly with various dietary components, and in particular certain minerals such as $\mathrm{Ca}$ and $\mathrm{Zn}$, and thus reduces their availability to humans and animals (Bohn et al., 2008). 
Although reports on contents of minerals in corn (Watson, 1987) and DDGS (Spiehs et al., 2002) are readily available, data on their changes during the entire dry-grind process, from corn to DDGS, are limited. Data on different forms of $\mathrm{P}$ in corn and DDGS, as well as their changes during dry-grind processing, are even scarcer. Rausch et al. (2005) monitored total P concentration in corn wet-milling streams but did not study dry-grind streams. Belyea et al. (2006) was the first to examine changes in mineral composition during the dry-grind process within a plant and the variation among ethanol plants, but they did not measure different forms of P. Noureddini et al. (2009) analyzed total P in different streams of both dry-grind and wet milling operations, but for inorganic $\mathrm{P}$ and phytate $\mathrm{P}$, only whole stillage and its centrifuged fractions were measured. The objective of this study was to determine concentrations of minerals as well as levels of different forms of $P$ in all possible streams of the dry-grind process, from corn to DDGS, and also variation among ethanol plants. Changes in gross composition, amino acid profile, and functional lipid profiles during the dry-grind process of corn into ethanol were reported elsewhere (Han and Liu, 2010; Moreau et al., 2010). Information on chemical changes during the entire dry-grind process can help us better understand the reasons for nutrient variation and develop strategies to modify individual processing steps for balancing nutrients in DDGS.

\section{Methods}

\subsection{Materials}

Samples of ground corn, intermediate streams, and DDGS were obtained from three dry-grind ethanol plants (numbered 1-3) located in the state of Iowa, USA. The intermediate streams included cooked slurry, liquefied mash, fermented mash (commonly known as beer), whole stillage, thin stillage, condensed distillers solubles (also known as syrup), and distillers wet grains. So, each sample set consisted of nine fractions. All samples were frozen after collection for transportation and storage. Just before chemical analysis, they were freeze-dried, and then ground into powder by a Cyclone Sample Mill (UDY Corp, Forth Collins, CO) with a built-in sieve of $0.5 \mathrm{~mm}$ round openings. However, dried thin stillage and syrup samples were ground by a small coffee grinder due to high oil content and soft texture.

\subsection{Chemical analysis}

All samples were analyzed for contents of moisture, total $\mathrm{P}$, phytate $\mathrm{P}$, inorganic $\mathrm{P}$, and 19 minerals ( $\mathrm{Ar}, \mathrm{Ba}, \mathrm{Ca}, \mathrm{Cd}, \mathrm{Co}, \mathrm{Cr}, \mathrm{Cu}$, $\mathrm{Fe}, \mathrm{K}, \mathrm{Mg}, \mathrm{Mn}, \mathrm{Mo}, \mathrm{Na}, \mathrm{Ni}, \mathrm{P}, \mathrm{Pb}, \mathrm{S}, \mathrm{V}$, and $\mathrm{Zn}$ ). Standard quality control measures, including blanks, check standards, reference materials, and duplicates, were used for all chemical analyses.

Moisture content was determined according to AOAC methods (AOAC, 2002), and used for converting concentrations of other components into a dry matter basis. Mineral elements were determined with a Perkin-Elmer Optima 3200 ICP-OES (inductively coupled plasma-optical emission spectrometer) to quantify constituents in an aqueous solution following nitric acid digestion of the samples. Total P, phytate $\mathrm{P}$, and inorganic $\mathrm{P}$ were measured by the methods described in Liu et al. (2007). Briefly, total P was determined after wet-ashing aliquots of samples $(150 \mathrm{mg}$ ) and colorimetric assay of $\mathrm{P}$ in the digests. Inorganic $\mathrm{P}$ was determined colorimetrically after extraction of tissue samples $(0.5 \mathrm{~g})$ in $12 \%(\mathrm{w} / \mathrm{v})$ trichloracetic acid (TCA) and $25 \mathrm{mM} \mathrm{MgCl}_{2}$, according to the method of Chen et al. (1956). For phytate P, aliquots of samples ( $0.5 \mathrm{~g}$ ) were extracted in $0.4 \mathrm{M} \mathrm{HCl}$ and $0.7 \mathrm{M} \mathrm{Na}_{2} \mathrm{SO}_{4}$. Phytate P was obtained as a ferric precipitate, wet-ashed, and assayed for $\mathrm{P}$ as in the total $\mathrm{P}$ analysis. All P-containing fractions are expressed as their $\mathrm{P}$ (atomic weight 31 ) content to facilitate comparisons. The rest of $\mathrm{P}$ was calculated by subtracting the sum of phytate $\mathrm{P}$ and inorganic P from total P.

\subsection{Data analysis and statistical treatment}

Data were analyzed with the JMP software, version 5 (JMP, a business unit of SAS, Cary, NC) for analysis of variance (ANOVA) based on a randomized block model in order to determine the effects of different processing steps (types of fractions) and ethanol plants (Plant No.). The Tukey's HSD (honestly significant difference) test was conducted for pair-wise comparisons when ANOVA showed a significant effect at $p<0.05$.

\section{Results and discussion}

\subsection{Changes in mineral concentrations}

Among the 19 minerals measured, 10 of them, including Ar, Ba, $\mathrm{Cd}, \mathrm{Co}, \mathrm{Cr}, \mathrm{Mo}, \mathrm{Na}, \mathrm{Ni}, \mathrm{Pb}$, and $\mathrm{V}$, had contents near or less than their detection limits based on the ICP method; data for these 10 minerals are not presented. In ground corn, major minerals, which had concentrations in the $\mathrm{mg} / \mathrm{g}$ range, were $\mathrm{P}, \mathrm{K}, \mathrm{Mg}, \mathrm{S}$, and $\mathrm{Ca}$; while minor minerals, which had concentrations in the $\mu \mathrm{g} / \mathrm{g}$ range, were $\mathrm{Zn}, \mathrm{Na}, \mathrm{Fe}, \mathrm{Mn}$, and $\mathrm{Cu}$ (Table 1). All mineral contents increased from ground corn to cooked slurry. The increase was most likely due to recycling a portion of thin stillage as cooking liquor since the contents of minerals in thin stillage were much higher than ground corn. Liquefication did not cause further change in mineral composition. However, upon fermentation, all nutrients increased dramatically. The increase was mainly due to depletion of starch which was fermented into ethanol and carbon dioxide. Distillation caused little changes in mineral composition. Upon centrifugation, more minerals went to the liquid fraction (thin stillage) than the solid fraction (distiller wet grains). When distiller wet grains and condensed solubles were mixed and dried to form DDGS, the composition was averaged, and became closer to but significantly less than that of the whole stillage. Again, recycling a portion of thin stillage for use as cooking liquor explains why the mineral composition in DDGS was less than that of whole stillage since a portion of minerals was channeled into front streams.

\subsection{Fold of increase over ground corn in mineral concentrations}

As just discussed, compared with ground corn, all minerals increased in concentrations in down stream products (Table 1 ), but the extent of the increase varied greatly with individual streams and minerals. This observation becomes particularly evident when the increase in mineral concentrations in each downstream fraction is expressed as "fold of increase" over ground corn (Table 2). The term is defined as the ratio in measured concentration of an attribute between a downstream product and corn". In general, the majority of minerals, including $\mathrm{P}, \mathrm{K}, \mathrm{Mg}, \mathrm{Cu}, \mathrm{Fe}, \mathrm{Mn}$, and $\mathrm{Zn}$, followed a similar pattern in fold of increase as the process advanced. Concentrations of these nutrients increased about 1.5 fold or less in the steps prior to fermentation. Upon fermentation, the increase was 3-4 folds. The values remained unchanged during distillation. Centrifuge brought about 4.5-7.4 fold of increase in thin stillage, but only $1.5-2.5$ fold of increase in distillers wet grains. More importantly, in the final product (DDGS), concentrations of these minerals all had nearly 3-fold increase. This 3-fold increase matches what is expected in DDGS since approximately two-thirds of the weight of incoming corn is converted into carbon dioxide and ethanol during fermentation (Bothast and Schlicher, 2005). 
Table 1

Changes in mineral concentrations during dry-grind processing of corn into ethanol at three plants. ${ }^{\mathrm{A}}$

\begin{tabular}{|c|c|c|c|c|c|c|c|c|c|c|c|}
\hline Fraction name & Plant No. & $\begin{array}{l}\mathrm{K} \\
(\mathrm{mg} / \mathrm{g})\end{array}$ & $\begin{array}{l}\mathrm{P} \\
(\mathrm{mg} / \mathrm{g})\end{array}$ & $\begin{array}{l}\mathrm{Mg} \\
(\mathrm{mg} / \mathrm{g})\end{array}$ & $\begin{array}{l}\mathrm{S} \\
(\mathrm{mg} / \mathrm{g})\end{array}$ & $\begin{array}{l}\mathrm{Na} \\
(\mathrm{mg} / \mathrm{g})\end{array}$ & $\begin{array}{l}\mathrm{Ca} \\
(\mathrm{mg} / \mathrm{g})\end{array}$ & $\begin{array}{l}\mathrm{Fe} \\
(\mu \mathrm{g} / \mathrm{g})\end{array}$ & $\begin{array}{l}\mathrm{Zn} \\
(\mu \mathrm{g} / \mathrm{g})\end{array}$ & $\begin{array}{l}\mathrm{Mn} \\
(\mu \mathrm{g} / \mathrm{g})\end{array}$ & $\begin{array}{l}\mathrm{Cu} \\
(\mu \mathrm{g} / \mathrm{g})\end{array}$ \\
\hline Ground $\operatorname{corn}^{\mathrm{C}}$ & $\begin{array}{l}1 \\
2 \\
3 \\
\text { Mean }^{\mathrm{B}} \\
\mathrm{CV}(\%)^{\mathrm{C}}\end{array}$ & $\begin{array}{l}4.10 \mathrm{x} \\
3.83 \mathrm{x} \\
3.83 \mathrm{x} \\
3.92 \mathrm{~g} \\
3.92\end{array}$ & $\begin{array}{l}3.54 \mathrm{x} \\
3.24 \mathrm{y} \\
3.38 \mathrm{xy} \\
3.39 \mathrm{~g} \\
4.33\end{array}$ & $\begin{array}{l}1.31 \mathrm{x} \\
1.18 \mathrm{y} \\
1.14 \mathrm{y} \\
1.21 \mathrm{f} \\
7.13\end{array}$ & $\begin{array}{l}1.08 \mathrm{x} \\
1.07 \mathrm{x} \\
1.04 \mathrm{x} \\
1.07 \mathrm{f} \\
1.91\end{array}$ & $\begin{array}{l}0.01 \mathrm{x} \\
0.01 \mathrm{x} \\
0.01 \mathrm{x} \\
0.01 \mathrm{~h} \\
0.00\end{array}$ & $\begin{array}{l}0.07 \mathrm{x} \\
0.09 \mathrm{x} \\
0.05 \mathrm{x} \\
0.07 \mathrm{~g} \\
28.11\end{array}$ & $\begin{array}{l}10.25 \mathrm{x} \\
8.78 \mathrm{xy} \\
8.01 \mathrm{y} \\
9.02 \mathrm{~d} \\
12.62\end{array}$ & $\begin{array}{l}23.42 \mathrm{x} \\
21.23 \mathrm{x} \\
22.36 \mathrm{x} \\
22.34 \mathrm{~g} \\
4.91\end{array}$ & $\begin{array}{l}5.36 \mathrm{x} \\
5.19 \mathrm{x} \\
5.42 \mathrm{x} \\
5.32 \mathrm{~h} \\
2.26\end{array}$ & $\begin{array}{l}1.92 \mathrm{x} \\
1.83 \mathrm{x} \\
2.01 \mathrm{x} \\
1.92 \mathrm{f} \\
4.69\end{array}$ \\
\hline Cooked slurry & $\begin{array}{l}1 \\
2 \\
3 \\
\text { Mean }^{\text {B }} \\
\text { CV }(\%)\end{array}$ & $\begin{array}{l}6.30 \\
5.92 \\
5.20 \\
5.80 \text { e } \\
9.60\end{array}$ & $\begin{array}{l}5.09 \\
4.69 \\
4.43 \\
4.74 \text { ef } \\
7.02\end{array}$ & $\begin{array}{l}1.90 \\
1.73 \\
1.55 \\
1.73 \mathrm{e} \\
10.17\end{array}$ & $\begin{array}{l}2.07 \\
1.63 \\
1.55 \\
1.75 \mathrm{e} \\
16.08\end{array}$ & $\begin{array}{l}0.93 \\
0.88 \\
0.70 \\
0.83 \mathrm{~g} \\
14.34\end{array}$ & $\begin{array}{l}0.14 \\
0.19 \\
0.12 \\
0.15 \mathrm{f} \\
23.70\end{array}$ & $\begin{array}{l}13.41 \\
9.59 \\
11.42 \\
11.47 \mathrm{~cd} \\
16.65\end{array}$ & $\begin{array}{l}30.41 \\
26.02 \\
26.27 \\
27.57 \mathrm{f} \\
8.94\end{array}$ & $\begin{array}{l}8.14 \\
7.30 \\
6.78 \\
7.41 \mathrm{~g} \\
9.27\end{array}$ & $\begin{array}{l}2.38 \\
2.19 \\
2.37 \\
2.31 \mathrm{f} \\
4.47\end{array}$ \\
\hline Liquefied mash & $\begin{array}{l}1 \\
2 \\
3 \\
\text { Mean }^{\mathrm{B}} \\
\text { CV }(\%)\end{array}$ & $\begin{array}{l}6.15 \\
5.85 \\
4.95 \\
5.65 \text { ef } \\
11.01\end{array}$ & $\begin{array}{l}4.44 \\
4.62 \\
4.34 \\
4.47 \mathrm{f} \\
3.22\end{array}$ & $\begin{array}{l}1.81 \\
1.75 \\
1.44 \\
1.66 \mathrm{e} \\
11.95\end{array}$ & $\begin{array}{l}1.81 \\
1.59 \\
2.37 \\
1.92 \mathrm{e} \\
20.96\end{array}$ & $\begin{array}{l}0.85 \\
0.86 \\
0.70 \\
0.80 \mathrm{~g} \\
11.32\end{array}$ & $\begin{array}{l}0.15 \\
0.19 \\
0.12 \\
0.15 \mathrm{f} \\
24.20\end{array}$ & $\begin{array}{l}9.97 \\
9.27 \\
7.62 \\
8.95 \mathrm{~d} \\
13.52\end{array}$ & $\begin{array}{l}25.32 \\
25.93 \\
25.82 \\
25.69 \mathrm{fg} \\
1.27\end{array}$ & $\begin{array}{l}6.98 \\
7.29 \\
6.77 \\
7.01 \mathrm{~g} \\
3.73\end{array}$ & $\begin{array}{l}2.07 \\
2.13 \\
2.36 \\
2.18 \mathrm{f} \\
6.96\end{array}$ \\
\hline Fermented mash & $\begin{array}{l}1 \\
2 \\
3 \\
\text { Mean }^{\text {B }} \\
\text { CV }(\%)\end{array}$ & $\begin{array}{l}16.51 \\
16.35 \\
16.13 \\
16.33 \mathrm{c} \\
1.16\end{array}$ & $\begin{array}{l}13.09 \\
13.53 \\
13.35 \\
13.32 \mathrm{c} \\
1.66\end{array}$ & $\begin{array}{l}4.84 \\
4.79 \\
4.51 \\
4.71 \mathrm{c} \\
3.83\end{array}$ & $\begin{array}{l}8.03 \\
6.20 \\
7.62 \\
7.28 \mathrm{~b} \\
13.15\end{array}$ & $\begin{array}{l}2.50 \\
2.99 \\
3.95 \\
3.15 \mathrm{~d} \\
23.37\end{array}$ & $\begin{array}{l}0.38 \\
0.55 \\
0.46 \\
0.46 \mathrm{c} \\
19.18\end{array}$ & $\begin{array}{l}20.49 \\
19.72 \\
26.15 \\
22.12 \text { b } \\
15.86\end{array}$ & $\begin{array}{l}72.28 \\
75.57 \\
78.42 \\
75.42 \mathrm{c} \\
4.07\end{array}$ & $\begin{array}{l}19.35 \\
20.30 \\
21.14 \\
20.26 \mathrm{~d} \\
4.41\end{array}$ & $\begin{array}{l}6.26 \\
6.20 \\
7.29 \\
6.58 \mathrm{c} \\
9.28\end{array}$ \\
\hline Whole stillage & $\begin{array}{l}1 \\
2 \\
3 \\
\text { Mean }^{\text {B }} \\
\text { CV }(\%)\end{array}$ & $\begin{array}{l}17.04 \\
16.96 \\
16.69 \\
16.90 \mathrm{c} \\
1.09\end{array}$ & $\begin{array}{l}12.58 \\
13.01 \\
13.91 \\
13.16 \mathrm{c} \\
5.17\end{array}$ & $\begin{array}{l}5.19 \\
4.92 \\
4.73 \\
4.95 \mathrm{c} \\
4.73\end{array}$ & $\begin{array}{l}8.16 \\
6.62 \\
7.84 \\
7.54 \mathrm{~b} \\
10.81\end{array}$ & $\begin{array}{l}2.64 \\
5.20 \\
4.23 \\
4.02 \mathrm{c} \\
32.20\end{array}$ & $\begin{array}{l}0.40 \\
0.56 \\
0.47 \\
0.48 \mathrm{c} \\
16.77\end{array}$ & $\begin{array}{l}20.37 \\
22.65 \\
24.48 \\
22.50 \mathrm{~b} \\
9.15\end{array}$ & $\begin{array}{l}73.99 \\
75.80 \\
79.01 \\
76.26 \mathrm{c} \\
3.34\end{array}$ & $\begin{array}{l}19.47 \\
23.76 \\
21.70 \\
21.64 \mathrm{c} \\
9.91\end{array}$ & $\begin{array}{l}6.00 \\
6.05 \\
7.34 \\
6.47 \mathrm{c} \\
11.77\end{array}$ \\
\hline Thin stillage & $\begin{array}{l}1 \\
2 \\
3 \\
\text { Mean }^{B} \\
\text { CV }(\%)\end{array}$ & $\begin{array}{l}33.72 \\
30.98 \\
32.10 \\
32.26 \mathrm{a} \\
4.27\end{array}$ & $\begin{array}{l}23.51 \\
22.21 \\
25.68 \\
23.80 \text { a } \\
7.37\end{array}$ & $\begin{array}{l}9.58 \\
8.65 \\
8.70 \\
8.97 \mathrm{a} \\
5.82\end{array}$ & $\begin{array}{l}11.92 \\
7.77 \\
11.09 \\
10.26 \text { a } \\
21.40\end{array}$ & $\begin{array}{l}5.15 \\
8.59 \\
8.11 \\
7.28 \text { a } \\
25.57\end{array}$ & $\begin{array}{l}0.67 \\
0.84 \\
0.78 \\
0.76 \mathrm{a} \\
10.79\end{array}$ & $\begin{array}{l}29.89 \\
29.26 \\
36.77 \\
31.97 \text { a } \\
13.02\end{array}$ & $\begin{array}{l}104.00 \\
91.73 \\
98.04 \\
97.92 \mathrm{a} \\
6.27\end{array}$ & $\begin{array}{l}34.72 \\
30.97 \\
35.60 \\
33.76 \text { a } \\
7.29\end{array}$ & $\begin{array}{l}7.24 \\
7.07 \\
10.74 \\
8.35 \mathrm{a} \\
24.81\end{array}$ \\
\hline Distillers solubles & $\begin{array}{l}1 \\
2 \\
3 \\
\text { Mean }^{B} \\
\text { CV }(\%)\end{array}$ & $\begin{array}{l}29.14 \\
29.99 \\
32.16 \\
30.43 \text { b } \\
5.11\end{array}$ & $\begin{array}{l}19.90 \\
20.94 \\
24.29 \\
21.71 \text { b } \\
10.57\end{array}$ & $\begin{array}{l}8.28 \\
7.98 \\
8.18 \\
8.15 \text { a } \\
1.89\end{array}$ & $\begin{array}{l}12.25 \\
7.75 \\
11.17 \\
10.39 \text { a } \\
22.59\end{array}$ & $\begin{array}{l}4.83 \\
8.09 \\
8.06 \\
6.99 \text { b } \\
26.75\end{array}$ & $\begin{array}{l}0.63 \\
0.70 \\
0.65 \\
0.66 \mathrm{~b} \\
5.92\end{array}$ & $\begin{array}{l}38.35 \\
28.88 \\
28.94 \\
32.06 \text { a } \\
17.00\end{array}$ & $\begin{array}{l}93.99 \\
78.64 \\
68.80 \\
80.48 \text { b } \\
15.78\end{array}$ & $\begin{array}{l}30.14 \\
24.89 \\
25.82 \\
26.95 \text { b } \\
10.38\end{array}$ & $\begin{array}{l}6.34 \\
6.79 \\
10.52 \\
7.88 \text { b } \\
29.16\end{array}$ \\
\hline Wet grains & $\begin{array}{l}1 \\
2 \\
3 \\
\text { Mean }^{B} \\
\text { CV }(\%)\end{array}$ & $\begin{array}{l}4.22 \\
4.52 \\
4.42 \\
4.38 \mathrm{fg} \\
3.56\end{array}$ & $\begin{array}{l}5.38 \\
5.42 \\
4.94 \\
5.24 \text { e } \\
5.07\end{array}$ & $\begin{array}{l}1.45 \\
1.63 \\
1.48 \\
1.52 \mathrm{e} \\
6.53\end{array}$ & $\begin{array}{l}5.37 \\
4.79 \\
5.07 \\
5.07 \mathrm{~d} \\
5.74\end{array}$ & $\begin{array}{l}0.65 \\
1.42 \\
1.15 \\
1.07 \mathrm{f} \\
36.17\end{array}$ & $\begin{array}{l}0.15 \\
0.30 \\
0.18 \\
0.21 \mathrm{e} \\
40.00\end{array}$ & $\begin{array}{l}14.65 \\
17.35 \\
14.67 \\
15.56 \mathrm{c} \\
10.00\end{array}$ & $\begin{array}{l}49.60 \\
55.75 \\
61.03 \\
55.46 \text { e } \\
10.32\end{array}$ & $\begin{array}{l}7.40 \\
11.57 \\
9.37 \\
9.45 \mathrm{f} \\
22.07\end{array}$ & $\begin{array}{l}4.75 \\
5.15 \\
4.71 \\
4.87 \text { e } \\
5.05\end{array}$ \\
\hline $\mathrm{DDGS}^{\mathrm{C}}$ & $\begin{array}{l}1 \\
2 \\
3 \\
\text { Mean }^{\mathrm{B}} \\
\text { CV }(\%)\end{array}$ & $\begin{array}{l}12.42 \mathrm{x} \\
10.72 \mathrm{y} \\
11.18 \mathrm{y} \\
11.44 \mathrm{~d} \\
7.66\end{array}$ & $\begin{array}{l}10.07 \\
9.68 \\
10.18 \\
9.98 \mathrm{~d} \\
2.62\end{array}$ & $\begin{array}{l}3.63 \mathrm{x} \\
3.48 \mathrm{y} \\
3.24 \mathrm{z} \\
3.45 \mathrm{~d} \\
5.79\end{array}$ & $\begin{array}{l}7.94 \mathrm{x} \\
6.03 \mathrm{y} \\
6.51 \mathrm{y} \\
6.83 \mathrm{c} \\
14.56\end{array}$ & $\begin{array}{l}2.16 \mathrm{y} \\
2.78 \mathrm{x} \\
2.94 \mathrm{x} \\
2.63 \mathrm{e} \\
15.56\end{array}$ & $\begin{array}{l}0.31 \mathrm{y} \\
0.48 \mathrm{x} \\
0.32 \mathrm{y} \\
0.37 \mathrm{~d} \\
26.02\end{array}$ & $\begin{array}{l}20.28 \mathrm{y} \\
26.63 \mathrm{x} \\
17.52 \mathrm{y} \\
21.47 \mathrm{~b} \\
21.75\end{array}$ & $\begin{array}{l}63.36 \mathrm{x} \\
67.28 \mathrm{x} \\
64.82 \mathrm{x} \\
65.15 \mathrm{~d} \\
3.04\end{array}$ & $\begin{array}{l}14.88 \mathrm{y} \\
17.98 \mathrm{x} \\
14.57 \mathrm{y} \\
15.81 \mathrm{e} \\
11.93\end{array}$ & $\begin{array}{l}5.01 \mathrm{y} \\
5.57 \mathrm{xy} \\
6.07 \mathrm{x} \\
5.55 \mathrm{~d} \\
9.52\end{array}$ \\
\hline Mean of fractions ${ }^{C}$ & $\begin{array}{l}1 \\
2 \\
3\end{array}$ & $\begin{array}{l}14.40 \mathrm{x} \\
13.90 \mathrm{x} \\
14.07 \mathrm{x}\end{array}$ & $\begin{array}{l}10.84 \mathrm{y} \\
10.82 \mathrm{y} \\
11.61 \mathrm{x}\end{array}$ & $\begin{array}{l}4.22 \mathrm{x} \\
4.01 \mathrm{y} \\
3.88 \mathrm{z}\end{array}$ & $\begin{array}{l}6.52 \mathrm{x} \\
4.83 \mathrm{z} \\
6.03 \mathrm{~b}\end{array}$ & $\begin{array}{l}2.19 \mathrm{z} \\
3.43 \mathrm{x} \\
3.32 \mathrm{y}\end{array}$ & $\begin{array}{l}0.32 \mathrm{z} \\
0.43 \mathrm{x} \\
0.35 \mathrm{y}\end{array}$ & $\begin{array}{l}19.74 \mathrm{x} \\
19.51 \mathrm{x} \\
19.51 \mathrm{x}\end{array}$ & $\begin{array}{l}59.60 \mathrm{x} \\
57.55 \mathrm{x} \\
58.29 \mathrm{x}\end{array}$ & $\begin{array}{l}16.27 \mathrm{x} \\
16.58 \mathrm{x} \\
16.35 \mathrm{x}\end{array}$ & $\begin{array}{l}4.66 \mathrm{y} \\
4.78 \mathrm{y} \\
5.93 \mathrm{x}\end{array}$ \\
\hline
\end{tabular}

${ }^{\mathrm{D}}$ Column means for each of 3 plants bearing different xyz letters differ significantly at $p<0.05$.

A Means of duplicate results, dry weight basis.

в Column means of three plants for each of nine fractions bearing different a-g letters differ significantly at $p<0.05$.

C $\mathrm{CV}=$ coefficient of variation (also known as relative standard deviation).

For these nutrients, we can reasonably assume that during the entire process, little is lost or added and the total amounts were conserved.

Careful examination of Table 2, however, reveals that the remaining minerals, including $\mathrm{Na}, \mathrm{S}, \mathrm{Ca}$, and $\mathrm{Fe}$, deviated significantly from the normal pattern in fold of increase observed for majority of elements, as just described. Among the four minerals, Na was most exceptional in its concentration change. In ground corn, its content was almost undetectable (less than $0.01 \mathrm{mg} / \mathrm{g}$ ). This value changed dramatically in the steps prior to fermentation. The Na content increased more than 80 folds over ground corn. In comparison, these same steps only brought about 1.5 fold of increase for many other minerals. Fermentation caused another significant change; more than 300 folds of increase in Na concentration over the ground corn. Further increase was noticed in thin stillage and its condensed form. In DDGS, there was about 260 fold of increase in Na concentration. The extremely high folds of increase in Na content can only be explained by addition of exogenous $\mathrm{Na}$ during the process. It is well known that ethanol producers frequently use $\mathrm{NaOH}$ to sanitize their process lines. They also use $\mathrm{NaOH}$ to adjust $\mathrm{pH}$ for optimum enzyme and yeast performance. In both practices Na can enter the processing stream and eventually end up in DDGS. $S$ is another mineral that had concentrations in the processing streams well in excess of the expected increase. It had 7.77-fold increase in concentration in DDGS over ground corn, as compared with the expected 3-fold increase. 
Table 2

Ratios of streams vs. ground corn (fold of increase) in mineral concentrations during dry-grind processing of corn into ethanol at three plants. ${ }^{\mathrm{A}}$

\begin{tabular}{|c|c|c|c|c|c|c|c|c|c|c|c|}
\hline Fraction name & Plant No. & $\mathrm{K}$ & $\mathrm{P}$ & $\mathrm{Mg}$ & $\mathrm{S}$ & $\mathrm{Na}$ & $\mathrm{Ca}$ & $\mathrm{Fe}$ & $\mathrm{Zn}$ & $\mathrm{Mn}$ & $\mathrm{Cu}$ \\
\hline \multirow[t]{4}{*}{ Ground corn } & 1 & 1.00 & 1.00 & 1.00 & 1.00 & 1.00 & 1.00 & 1.00 & 1.00 & 1.00 & 1.00 \\
\hline & 2 & 1.00 & 1.00 & 1.00 & 1.00 & 1.00 & 1.00 & 1.00 & 1.00 & 1.00 & 1.00 \\
\hline & 3 & 1.00 & 1.00 & 1.00 & 1.00 & 1.00 & 1.00 & 1.00 & 1.00 & 1.00 & 1.00 \\
\hline & Mean & $1.00 \mathrm{f}$ & $1.00 \mathrm{f}$ & $1.00 \mathrm{f}$ & $1.00 \mathrm{f}$ & $1.00 \mathrm{~h}$ & $1.00 \mathrm{e}$ & $1.00 \mathrm{c}$ & $1.00 \mathrm{e}$ & $1.00 \mathrm{~h}$ & $1.00 \mathrm{~d}$ \\
\hline \multirow[t]{4}{*}{ Cooked slurry } & 1 & 1.54 & 1.44 & 1.45 & 1.91 & 92.53 & 2.00 & 1.31 & 1.30 & 1.52 & 1.24 \\
\hline & 2 & 1.54 & 1.45 & 1.47 & 1.52 & 87.76 & 2.10 & 1.09 & 1.23 & 1.41 & 1.20 \\
\hline & 3 & 1.36 & 1.31 & 1.35 & 1.48 & 69.85 & 2.37 & 1.42 & 1.17 & 1.25 & 1.18 \\
\hline & Mean & $1.48 \mathrm{e}$ & $1.40 \mathrm{e}$ & $1.43 \mathrm{e}$ & $1.64 \mathrm{e}$ & $83.38 \mathrm{~g}$ & $2.13 \mathrm{~d}$ & $1.27 \mathrm{c}$ & $1.23 \mathrm{e}$ & $1.39 \mathrm{~g}$ & $1.21 \mathrm{~d}$ \\
\hline \multirow[t]{4}{*}{ Liquefied mash } & 1 & 1.50 & 1.26 & 1.38 & 1.67 & 84.74 & 2.09 & 0.97 & 1.08 & 1.30 & 1.08 \\
\hline & 2 & 1.53 & 1.42 & 1.48 & 1.48 & 86.26 & 2.05 & 1.06 & 1.22 & 1.41 & 1.17 \\
\hline & 3 & 1.29 & 1.28 & 1.26 & 2.27 & 69.82 & 2.24 & 0.95 & 1.15 & 1.25 & 1.17 \\
\hline & Mean & $1.44 \mathrm{e}$ & $1.32 \mathrm{e}$ & $1.37 \mathrm{e}$ & $1.80 \mathrm{e}$ & $80.28 \mathrm{~g}$ & $2.11 \mathrm{~d}$ & $0.99 \mathrm{c}$ & $1.15 \mathrm{e}$ & $1.32 \mathrm{~g}$ & $1.14 \mathrm{~d}$ \\
\hline \multirow[t]{4}{*}{ Fermented mash } & 1 & 4.03 & 3.70 & 3.70 & 7.40 & 250.45 & 5.23 & 2.00 & 3.09 & 3.61 & 3.27 \\
\hline & 2 & 4.27 & 4.17 & 4.06 & 5.78 & 298.74 & 5.97 & 2.25 & 3.56 & 3.91 & 3.39 \\
\hline & 3 & 4.22 & 3.95 & 3.94 & 7.30 & 394.92 & 8.77 & 3.26 & 3.51 & 3.90 & 3.63 \\
\hline & Mean & $4.17 \mathrm{c}$ & $3.94 \mathrm{c}$ & $3.89 \mathrm{c}$ & $6.82 \mathrm{bc}$ & $314.70 \mathrm{~d}$ & $6.40 \mathrm{~b}$ & $2.45 \mathrm{~b}$ & $3.38 \mathrm{~b}$ & $3.81 \mathrm{~d}$ & $3.43 \mathrm{~b}$ \\
\hline \multirow[t]{4}{*}{ Whole stillage } & 1 & 4.16 & 3.56 & 3.97 & 7.53 & 263.69 & 5.57 & 1.99 & 3.16 & 3.63 & 3.13 \\
\hline & 2 & 4.43 & 4.01 & 4.17 & 6.17 & 520.33 & 6.05 & 2.58 & 3.57 & 4.58 & 3.31 \\
\hline & 3 & 4.36 & 4.12 & 4.13 & 7.51 & 422.84 & 9.10 & 3.06 & 3.53 & 4.00 & 3.66 \\
\hline & Mean & $4.31 \mathrm{c}$ & $3.89 \mathrm{c}$ & $4.09 \mathrm{c}$ & $7.07 \mathrm{~b}$ & $402.29 \mathrm{c}$ & $6.62 \mathrm{~b}$ & $2.50 \mathrm{~b}$ & $3.41 \mathrm{~b}$ & $4.07 \mathrm{c}$ & $3.37 \mathrm{~b}$ \\
\hline \multirow[t]{4}{*}{ Thin stillage } & 1 & 8.23 & 6.65 & 7.32 & 11.00 & 515.19 & 9.37 & 2.91 & 4.44 & 6.47 & 3.78 \\
\hline & 2 & 8.08 & 6.85 & 7.33 & 7.24 & 859.08 & 9.03 & 3.33 & 4.32 & 5.97 & 3.87 \\
\hline & 3 & 8.39 & 7.61 & 7.60 & 10.62 & 811.21 & 14.93 & 4.59 & 4.38 & 6.57 & 5.35 \\
\hline & Mean & $8.23 \mathrm{a}$ & $7.03 \mathrm{a}$ & $7.41 \mathrm{a}$ & $9.62 \mathrm{a}$ & 728.49 a & $10.56 \mathrm{a}$ & $3.55 \mathrm{a}$ & $4.38 \mathrm{a}$ & $6.34 \mathrm{a}$ & $4.35 \mathrm{a}$ \\
\hline \multirow[t]{4}{*}{ Distillers solubles } & 1 & 7.12 & 5.63 & 6.33 & 11.30 & 483.41 & 8.72 & 3.74 & 4.01 & 5.62 & 3.31 \\
\hline & 2 & 7.83 & 6.46 & 6.77 & 7.22 & 809.20 & 7.58 & 3.29 & 3.71 & 4.80 & 3.72 \\
\hline & 3 & 8.41 & 7.20 & 7.14 & 10.70 & 805.83 & 12.44 & 3.61 & 3.08 & 4.77 & 5.24 \\
\hline & Mean & $7.77 \mathrm{~b}$ & $6.41 \mathrm{~b}$ & $6.73 \mathrm{~b}$ & $9.74 \mathrm{a}$ & $699.48 \mathrm{~b}$ & $9.13 \mathrm{a}$ & $3.55 \mathrm{a}$ & $3.60 \mathrm{~b}$ & $5.06 \mathrm{~b}$ & $4.11 \mathrm{a}$ \\
\hline \multirow[t]{4}{*}{ Wet grains } & 1 & 1.03 & 1.52 & 1.10 & 4.95 & 65.37 & 2.03 & 1.43 & 2.12 & 1.38 & 2.48 \\
\hline & 2 & 1.18 & 1.67 & 1.38 & 4.46 & 141.97 & 3.30 & 1.98 & 2.63 & 2.23 & 2.82 \\
\hline & 3 & 1.15 & 1.46 & 1.29 & 4.85 & 114.79 & 3.44 & 1.83 & 2.73 & 1.73 & 2.35 \\
\hline & Mean & $1.12 \mathrm{f}$ & $1.55 \mathrm{e}$ & $1.25 \mathrm{e}$ & $4.75 \mathrm{~d}$ & $107.38 \mathrm{f}$ & $2.91 \mathrm{~cd}$ & $1.72 \mathrm{bc}$ & $2.48 \mathrm{~d}$ & $1.77 \mathrm{f}$ & $2.54 \mathrm{c}$ \\
\hline \multirow[t]{4}{*}{ DDGS } & 1 & 3.03 & 2.85 & 2.78 & 7.33 & 216.39 & 4.36 & 1.98 & 2.71 & 2.77 & 2.62 \\
\hline & 2 & 2.80 & 2.99 & 2.95 & 5.62 & 278.32 & 5.20 & 3.03 & 3.17 & 3.47 & 3.05 \\
\hline & 3 & 2.92 & 3.02 & 2.83 & 6.23 & 293.63 & 6.07 & 2.19 & 2.90 & 2.69 & 3.02 \\
\hline & Mean & $2.92 \mathrm{~d}$ & $2.95 \mathrm{~d}$ & $2.85 \mathrm{~d}$ & $6.40 \mathrm{c}$ & $262.78 \mathrm{e}$ & $5.13 \mathrm{bc}$ & $2.38 \mathrm{~b}$ & $2.92 \mathrm{c}$ & $2.97 \mathrm{e}$ & $2.90 \mathrm{c}$ \\
\hline
\end{tabular}

A Column means of three plants for each of nine fractions bearing different letters differ significantly at $p<0.05$.

Presumably, increased S reflects the addition of S compounds (e.g. sulfuric acid) to adjust the $\mathrm{pH}$ for optimum enzyme activity during liquefaction or optimum yeast growth during fermentation. The third mineral that had unexpected increase in concentration in several processing streams was Ca. In DDGS, the fold of increase over corn exceeded 5. Presumably, a Ca compound might be added during the process. Fe was the only mineral that the fold of increase in its concentration of DDGS over corn was less than 3 (2.38, on average). The reason for this observation is unknown.

Previous studies also reported larger folds of increase in $\mathrm{Na}, \mathrm{S}$, and Ca concentrations in processing streams (including DDGS) over corn as compared to other minerals. Batal and Dale (2003) noticed that the content of most minerals in DDGS appeared generally consistent with a 3 fold of increase over corn but unusually larger ranges of values were noticed for Na and Ca. Belyea et al. (2006) reported that there were differences among streams for mean concentrations of many elements and that the concentrations of most elements in beer were three times of those in corn. However, in the same beer stream, both Na and S had unusually higher increases in concentrations than other minerals. They attributed this to addition of $\mathrm{Na}$ and $\mathrm{S}$ compounds during the dry-grind process. With regard to which stream product has the highest element concentration, Belyea et al. (2006) pointed to syrup, but the present study indicated thin stillage. The difference is apparently due to the fact that Belyea et al. (2006) did not include samples of certain key steps, including cooked slurry, liquefied mass and thin stillage, for their study.

\subsection{Changes in concentrations of different forms of $P$}

Among all the minerals in DDGS, P is an important element to consider. Thus, the second objective of this study was to identify the specific steps in the dry-grind ethanol process, which cause significant changes in different forms of P. As shown in Table 3, the changing pattern of $P$ profile during the dry-grind process generally resembled that of mineral composition. The contents of phytate $\mathrm{P}$, inorganic $\mathrm{P}$ and total $\mathrm{P}$ increased significantly from ground corn to cooked slurry. This increase was most likely due to recycling a fraction of thin stillage as cooking water, since thin stillage had very higher $\mathrm{P}$ levels. The contents of these three forms of $\mathrm{P}$ fluctuated slightly until fermentation. In contrast, the rest of P remained relatively unchanged from ground corn to fermentation. After fermentation, all four forms of $\mathrm{P}$ increased dramatically. These increases resulted mainly from depletion of starch in the mass. Distillation caused little change in P profile. Upon centrifuging whole stillage, the two resulting fractions exhibited a huge difference in P profile, with thin stillage having much higher contents for all forms of $\mathrm{P}$ than distiller wet grains. In fact, thin stillage had the highest levels of four forms of $P$ among all the samples measured. In contrast, distiller wet grains had a phytate P level similar to ground corn while the other three forms of P were only slightly higher than those in corn. Upon mixing the solubles with wet grains and drying the mixture into DDGS, the contents of all forms of $\mathrm{P}$ were brought back almost to the levels in whole stillage, but 
Table 3

Changes in various forms of phosphorus $(\mathrm{P})$ during dry-grind processing of corn into ethanol at three plants. ${ }^{\mathrm{A}, \mathrm{B}}$

\begin{tabular}{|c|c|c|c|c|c|c|c|c|}
\hline Fraction name & Plant No. & $\begin{array}{l}\text { Phy P } \\
(\mathrm{mg} / \mathrm{g})\end{array}$ & $\begin{array}{l}\text { Inorg P } \\
(\mathrm{mg} / \mathrm{g})\end{array}$ & $\begin{array}{l}\text { Rest of } \mathrm{P} \\
(\mathrm{mg} / \mathrm{g})\end{array}$ & $\begin{array}{l}\text { Total P } \\
(\mathrm{mg} / \mathrm{g})\end{array}$ & $\begin{array}{l}\text { Phy P/TP } \\
(\%)\end{array}$ & $\begin{array}{l}\text { Inorg } \mathrm{P} / \mathrm{TP} \\
(\%)\end{array}$ & $\begin{array}{l}\text { Rest P/TP } \\
(\%)\end{array}$ \\
\hline Ground $\operatorname{corn}^{\mathrm{D}}$ & $\begin{array}{l}1 \\
2 \\
3 \\
\text { Mean }^{\mathrm{C}} \\
\text { CV (\%) }\end{array}$ & $\begin{array}{l}2.22 \mathrm{x} \\
1.99 \mathrm{y} \\
2.15 \mathrm{xy} \\
2.12 \mathrm{~g} \\
5.69\end{array}$ & $\begin{array}{l}0.26 \mathrm{x} \\
0.23 \mathrm{y} \\
0.27 \mathrm{x} \\
0.26 \mathrm{f} \\
7.60\end{array}$ & $\begin{array}{l}0.52 \mathrm{x} \\
0.53 \mathrm{x} \\
0.49 \mathrm{x} \\
0.51 \mathrm{~d} \\
4.31\end{array}$ & $\begin{array}{l}3.01 \mathrm{x} \\
2.75 \mathrm{x} \\
2.90 \mathrm{x} \\
2.89 \mathrm{~g} \\
4.42\end{array}$ & $\begin{array}{l}73.97 \mathrm{x} \\
72.19 \mathrm{x} \\
74.11 \mathrm{x} \\
73.42 \mathrm{a} \\
1.46\end{array}$ & $\begin{array}{l}8.76 \mathrm{x} \\
8.47 \mathrm{x} \\
9.30 \mathrm{x} \\
8.84 \mathrm{f} \\
4.76\end{array}$ & $\begin{array}{l}17.27 \mathrm{x} \\
19.35 \mathrm{x} \\
16.59 \mathrm{x} \\
17.74 \mathrm{~b} \\
8.08\end{array}$ \\
\hline Cooked slurry & $\begin{array}{l}1 \\
2 \\
3 \\
\text { Mean }^{\mathrm{C}} \\
\text { CV (\%) }\end{array}$ & $\begin{array}{l}3.65 \\
3.19 \\
3.06 \\
3.30 \mathrm{f} \\
9.45\end{array}$ & $\begin{array}{l}0.61 \\
0.81 \\
0.55 \\
0.66 \text { e } \\
20.45\end{array}$ & $\begin{array}{l}0.52 \\
0.54 \\
0.68 \\
0.58 \mathrm{~d} \\
15.02\end{array}$ & $\begin{array}{l}4.79 \\
4.54 \\
4.30 \\
4.54 \text { ef } \\
5.46\end{array}$ & $\begin{array}{l}76.28 \\
70.30 \\
71.24 \\
72.61 \mathrm{a} \\
4.42\end{array}$ & $\begin{array}{l}12.82 \\
17.85 \\
12.86 \\
14.51 \mathrm{e} \\
19.93\end{array}$ & $\begin{array}{l}10.90 \\
11.85 \\
15.89 \\
12.88 \text { bc } \\
20.58\end{array}$ \\
\hline Liquefied mash & $\begin{array}{l}1 \\
2 \\
3 \\
\text { Meanc }^{\mathrm{C}} \\
\text { CV (\%) }\end{array}$ & $\begin{array}{l}3.13 \\
3.25 \\
3.09 \\
3.16 \mathrm{f} \\
2.69\end{array}$ & $\begin{array}{l}0.68 \\
0.71 \\
0.55 \\
0.65 \text { e } \\
12.72\end{array}$ & $\begin{array}{l}0.40 \\
0.54 \\
0.56 \\
0.50 \mathrm{~d} \\
18.08\end{array}$ & $\begin{array}{l}4.20 \\
4.50 \\
4.20 \\
4.30 \mathrm{f} \\
4.00\end{array}$ & $\begin{array}{l}74.48 \\
72.28 \\
73.40 \\
73.39 \mathrm{a} \\
1.50\end{array}$ & $\begin{array}{l}16.09 \\
15.79 \\
13.18 \\
15.02 \mathrm{e} \\
10.67\end{array}$ & $\begin{array}{l}9.43 \\
11.93 \\
13.42 \\
11.59 \mathrm{c} \\
17.38\end{array}$ \\
\hline Fermented mash & $\begin{array}{l}1 \\
2 \\
3 \\
\text { Mean }^{\mathrm{C}} \\
\text { CV }(\%)\end{array}$ & $\begin{array}{l}5.19 \\
5.42 \\
5.54 \\
5.38 \mathrm{~d} \\
3.26\end{array}$ & $\begin{array}{l}3.00 \\
2.73 \\
2.97 \\
2.90 \mathrm{c} \\
5.02\end{array}$ & $\begin{array}{l}2.80 \\
3.85 \\
3.51 \\
3.39 \mathrm{a} \\
15.80\end{array}$ & $\begin{array}{l}11.00 \\
12.00 \\
12.01 \\
11.67 \mathrm{c} \\
5.01\end{array}$ & $\begin{array}{l}47.23 \\
45.13 \\
46.13 \\
46.16 \mathrm{c} \\
2.28\end{array}$ & $\begin{array}{l}27.30 \\
22.78 \\
24.72 \\
24.93 \text { c } \\
9.09\end{array}$ & $\begin{array}{l}25.47 \\
32.09 \\
29.15 \\
28.9 a \\
11.48\end{array}$ \\
\hline Whole stillage & $\begin{array}{l}1 \\
2 \\
3 \\
\text { Mean }^{\mathrm{C}} \\
\text { CV }(\%)\end{array}$ & $\begin{array}{l}5.24 \\
5.50 \\
6.37 \\
5.71 \mathrm{c} \\
10.40\end{array}$ & $\begin{array}{l}2.95 \\
2.74 \\
3.28 \\
2.99 \mathrm{c} \\
9.11\end{array}$ & $\begin{array}{l}2.92 \\
3.28 \\
3.34 \\
3.18 \mathrm{a} \\
7.18\end{array}$ & $\begin{array}{l}11.11 \\
11.52 \\
12.99 \\
11.88 \mathrm{c} \\
8.35\end{array}$ & $\begin{array}{l}47.18 \\
47.74 \\
49.05 \\
47.99 \mathrm{c} \\
2.00\end{array}$ & $\begin{array}{l}26.54 \\
23.78 \\
25.24 \\
25.19 \mathrm{c} \\
5.47\end{array}$ & $\begin{array}{l}26.28 \\
28.47 \\
25.71 \\
26.82 \mathrm{a} \\
5.43\end{array}$ \\
\hline Thin stillage & $\begin{array}{l}1 \\
2 \\
3 \\
\text { Mean }^{\mathrm{C}} \\
\mathrm{CV}(\%)\end{array}$ & $\begin{array}{l}9.20 \\
9.40 \\
11.01 \\
9.87 \mathrm{a} \\
10.03\end{array}$ & $\begin{array}{l}5.63 \\
6.62 \\
6.62 \\
6.29 \mathrm{~b} \\
9.05\end{array}$ & $\begin{array}{l}3.86 \\
2.99 \\
4.03 \\
3.63 \mathrm{a} \\
15.43\end{array}$ & $\begin{array}{l}18.69 \\
19.01 \\
21.65 \\
19.78 \text { a } \\
8.22\end{array}$ & $\begin{array}{l}49.21 \\
49.47 \\
50.83 \\
49.84 \mathrm{~b} \\
1.74\end{array}$ & $\begin{array}{l}30.15 \\
34.81 \\
30.56 \\
31.84 \text { b } \\
8.11\end{array}$ & $\begin{array}{l}20.64 \\
15.72 \\
18.62 \\
18.33 \text { b } \\
13.51\end{array}$ \\
\hline Distillers solubles & $\begin{array}{l}1 \\
2 \\
3 \\
\text { Mean } \\
\text { CV }(\%)\end{array}$ & $\begin{array}{l}7.67 \\
8.23 \\
9.96 \\
8.62 \text { b } \\
13.83\end{array}$ & $\begin{array}{l}6.08 \\
6.82 \\
7.42 \\
6.77 \text { a } \\
9.88\end{array}$ & $\begin{array}{l}3.25 \\
3.45 \\
3.38 \\
3.36 \mathrm{a} \\
2.93\end{array}$ & $\begin{array}{l}17.01 \\
18.50 \\
20.76 \\
18.75 \mathrm{~b} \\
10.07\end{array}$ & $\begin{array}{l}45.11 \\
44.53 \\
48.00 \\
45.88 \mathrm{~cd} \\
4.06\end{array}$ & $\begin{array}{l}35.76 \\
36.87 \\
35.74 \\
36.12 \text { a } \\
1.80\end{array}$ & $\begin{array}{l}19.13 \\
18.60 \\
16.26 \\
18,00 \mathrm{~b} \\
8.47\end{array}$ \\
\hline Distillers wet grains & $\begin{array}{l}1 \\
2 \\
3 \\
\text { Mean } \\
\text { CV }(\%)\end{array}$ & $\begin{array}{l}2.32 \\
2.59 \\
2.22 \\
2.38 \mathrm{~g} \\
8.18\end{array}$ & $\begin{array}{l}1.07 \\
0.91 \\
0.78 \\
0.92 \mathrm{~d} \\
15.92\end{array}$ & $\begin{array}{l}1.16 \\
1.72 \\
1.34 \\
1.41 \mathrm{c} \\
20.37\end{array}$ & $\begin{array}{l}4.55 \\
5.23 \\
4.34 \\
4.71 \mathrm{e} \\
9.81\end{array}$ & $\begin{array}{l}51.01 \\
49.63 \\
51.03 \\
50.56 \mathrm{~b} \\
1.59\end{array}$ & $\begin{array}{l}23.59 \\
17.48 \\
17.99 \\
19.69 \mathrm{~d} \\
17.24\end{array}$ & $\begin{array}{l}25.40 \\
32.89 \\
30.98 \\
29.76 \text { a } \\
13.09\end{array}$ \\
\hline $\mathrm{DDGS}^{\mathrm{D}}$ & $\begin{array}{l}1 \\
2 \\
3 \\
\text { Mean }^{\mathrm{C}} \\
\text { CV }(\%)\end{array}$ & $\begin{array}{l}3.76 \mathrm{x} \\
3.79 \mathrm{x} \\
3.88 \mathrm{x} \\
3.81 \mathrm{e} \\
1.67\end{array}$ & $\begin{array}{l}3.04 \mathrm{x} \\
2.56 \mathrm{y} \\
2.64 \mathrm{y} \\
2.75 \mathrm{c} \\
9.34\end{array}$ & $\begin{array}{l}1.76 \mathrm{x} \\
2.01 \mathrm{x} \\
2.76 \mathrm{x} \\
2.17 \mathrm{~b} \\
24.07\end{array}$ & $\begin{array}{l}8.56 \mathrm{y} \\
8.35 \mathrm{y} \\
9.28 \mathrm{x} \\
8.73 \mathrm{~d} \\
5.60\end{array}$ & $\begin{array}{l}43.90 \mathrm{x} \\
45.39 \mathrm{x} \\
41.82 \mathrm{x} \\
43.70 \mathrm{~d} \\
4.11\end{array}$ & $\begin{array}{l}35.55 \mathrm{x} \\
30.72 \mathrm{y} \\
28.45 \mathrm{y} \\
31.57 \mathrm{~b} \\
11.48\end{array}$ & $\begin{array}{l}20.55 \mathrm{y} \\
23.89 \mathrm{y} \\
29.73 \mathrm{x} \\
24.72 \mathrm{ab} \\
18.80\end{array}$ \\
\hline Mean of fractions ${ }^{\mathrm{D}}$ & $\begin{array}{l}1 \\
2 \\
3\end{array}$ & $\begin{array}{l}4.71 \mathrm{y} \\
4.82 \mathrm{y} \\
5.25 \mathrm{a}\end{array}$ & $\begin{array}{l}2.59 \mathrm{y} \\
2.68 \mathrm{xy} \\
2.79 \mathrm{x}\end{array}$ & $\begin{array}{l}1.91 \mathrm{y} \\
2.1 \mathrm{xy} \\
2.23 \mathrm{x}\end{array}$ & $\begin{array}{l}9.21 \mathrm{z} \\
9.60 \mathrm{y} \\
10.27 \mathrm{x}\end{array}$ & $\begin{array}{l}56.48 \times \\
55.18 \times \\
56.18 \times\end{array}$ & $\begin{array}{l}24.06 \mathrm{x} \\
23.17 \mathrm{y} \\
22.00 \mathrm{z}\end{array}$ & $\begin{array}{l}19.45 \mathrm{y} \\
21.64 \mathrm{xy} \\
21.82 \mathrm{x}\end{array}$ \\
\hline
\end{tabular}

\footnotetext{
${ }^{A}$ Means of duplicate results. Concentration is expressed on dry weight basis.

B $\mathrm{P}=$ phosphorus, $\mathrm{Phy}=$ phytate; Inorg $=$ inorganic, Rest $\mathrm{P}=$ the rest of $\mathrm{P}, \mathrm{TP}=$ total $\mathrm{P}, \mathrm{CV}=$ coefficient of variation.

C Column means of three plants for each of nine fractions bearing different a-g letters differ significantly at $p<0.05$.

D Column means for each of three plants bearing different xyz letters differ significantly at $p<0.05$.
}

generally less, apparently due to channeling some P into the front stream during recycling a portion of thin stillage as a backset. Understandably, the observed higher contents of all forms of $\mathrm{P}$ in DDGS than in distiller wet grains resulted mostly from addition of condensed distillers solubles.

Furthermore, we found some interesting trends with regard to contribution of each form of $\mathrm{P}$ toward the total $\mathrm{P}$ (Table 3 ). At the beginning stages of the process, up to fermentation, phytate $\mathrm{P}$ contributed about $73 \%$ toward total $\mathrm{P}$, while inorganic $\mathrm{P}$ and the rest of $P$ collectively contributed the remaining $27 \%$. Upon fermentation, contribution of phytate $\mathrm{P}$ toward total $\mathrm{P}$ decreased to about $46 \%$, while contribution by inorganic $\mathrm{P}$ and the rest of $\mathrm{P}$ increased to about $27 \%$ each (doubled). In the remaining processing streams, contribution of phytate $\mathrm{P}$ toward total $\mathrm{P}$ fluctuated slightly. However, inorganic P contributed more than the rest of $\mathrm{P}$ toward total $\mathrm{P}$ in thin stillage, while the opposite was observed in distiller wet grains. These observations indicate that: (1) upon fermentation phytate underwent some degradation, most likely due to the action of yeast phytase, (2) solubility of inorganic $\mathrm{P}$ (that is, phosphate $\mathrm{P}$ ) was higher than that of rest of $\mathrm{P}$. Thus, relatively speaking, more inorganic $P$ went into thin stillage than the rest of $\mathrm{P}$ during centrifugation, and (3) phytate $\mathrm{P}$ partitioning into the liquid fraction and solid fraction upon centrifugation was relatively proportional to that of total P. 
Note that the data of the total P by the wet chemistry method (Table 3$)$ were significantly lower $(p<0.05)$ than data of the element $P$ by the ICP method (Table 1 ), even though the two methods measured the same compounds. However, in barley samples, the two methods gave similar P values (Liu et al., 2007). Apparently, the complex matrix of DDGS might prevent complete extraction of total P by the wet method used. Since the present study focused on changing patterns during the process, and since the difference between the two methods for total $P$ were systematic, using data of total P by the wet method in Table 3 did not change any conclusion of the present study.

Noureddini et al. (2009) measured total P in several stream products of the dry-grind process, including whole corn, milled corn, whole stillage, wet grains, syrup (condensed distillers solubles), distillers wet grains with solubles, and DDGS. They found that syrup contained the highest total P concentration $(1.34 \%$, dry matter). The present study showed that syrup contained very high concentrations of total $\mathrm{P}$, with an average value of three plants as $1.88 \%$ (dry matter) for three plants, but thin stillage had the highest total $\mathrm{P}$ concentration (1.98\%). Noureddini et al. (2009) further showed that about 59\% of total P in whole stillage was phosphate $\mathrm{P}$, and attributed the remainder of $\mathrm{P}$ in whole stillage (41\%) as phytate P. They did not, however, consider the contribution by the rest of $P$ defined in this study. Furthermore, their HPLC analysis of this stream and its two centrifuged fractions did not reveal the presence of phytate. In contrast, the present study showed that about $48 \%$ of total $\mathrm{P}$ in whole stillage was phytate $\mathrm{P}$ and that phosphate $\mathrm{P}$ toward total $\mathrm{P}$ in whole stillage was only 25.19\%. The remaining $26.82 \%$ was contribution by the rest of $\mathrm{P}$. When the sum of contribution by both phosphate $P$ and the rest of $\mathrm{P}$ is collectively considered as nonphytate $\mathrm{P}$ contribution toward total $\mathrm{P}$, the present study showed that in DDGS the nonphytate $\mathrm{P}$ was $56.30 \%$ (Table 3 ). This value matches well with $54 \%$ reported by NRC (1994).

Moreover, in the study of Noureddini et al. (2009), several stream products, including cooked slurry, liquefied mash, fermented mash, and thin stillage, were not collected for analysis of total $\mathrm{P}$. The levels of phosphate $\mathrm{P}$ and phytate $\mathrm{P}$ were measured only in whole stillage, its liquid and solid fractions after laboratory centrifugation. The contribution by the rest of $\mathrm{P}$ was not accounted for in the stream and its fractions. Therefore, the present study is considered to be the first to document changes in all four forms of $\mathrm{P}$ during the entire dry-grind process from corn.

\subsection{Fold of increase in concentrations of different forms of $P$ over ground corn}

Although total $\mathrm{P}$ exhibited a similar pattern in fold of increase to those of most minerals, as previously discussed, the other three forms of P did not (Table 4). Phytate P increased about 1.5 fold in cooked slurry and liquefied mass, similar to that of other minerals. However, upon fermentation, phytate P increased only 2.54 fold, as compared with 3-4 folds for most other minerals. Concomitantly, fold of increase for inorganic $P$ concentration in these steps was much higher than that of most minerals: 2.58 fold in cooked slurry and 11.37 fold in fermented mash. In the final product (DDGS), the increase of phytate $P$ was 1.80 fold of corn, but inorganic P was 10.77 fold. Because of the dynamic changes of phytate $P$ and inorganic $\mathrm{P}$, the rest of $\mathrm{P}$ also showed some deviation from the normal pattern in fold of increase. The lower fold of increase in phytate $P$ concentration over ground corn and much higher fold of increase in inorganic $P$, as compared with other minerals, further indicates occurrence of phytate degradation during the dry-grind process. Phytase is widely distributed in plants and microorganisms, including grains and fermentation yeast (Wodzinski and Ullah, 1996). Based on Shetty et al. (2008), it is possible that phytate is hydrolyzed during steps prior to fermentation by endogenous phytase of feedstock (corn) and/or during fermentation by yeast phytase. It is also possible that non-enzymatic hydrolysis of phytate occurs under harsh processing conditions (heat and/or $\mathrm{pH}$ changes) during the dry-grind process. However, since the present study showed that only during fermentation were dramatic changes in phytate $\mathrm{P}$ and inorganic $\mathrm{P}$ were observed in term of \% relative to total $\mathrm{P}$ (Table 3 ) and fold of increase over corn (Table 4), activity of yeast phytase is most likely the major route for phytate degradation.

For years, bioavailability of $\mathrm{P}$ in DDGS has been repeatedly shown to be significantly higher than that in corn (Amezcua et al., 2004; Pedersen et al., 2007). In one report for nutritional requirements of swine (NRC, 1998), the relative availability of $P$ in corn was $14 \%$. This value increased to $77 \%$ for DDGS produced from corn. Phytate degradation during the dry-grind process, as supported by (1) observed decrease in \% phytate P and concomitant increase in \% inorganic $\mathrm{P}$, relative to total $\mathrm{P}$, during fermentation (Table 3), and (2) observed varying fold of increase over corn in concentrations of different forms of P (Table 4), would account for improved $\mathrm{P}$ bioavailability in DDGS over corn. Most of the $\mathrm{P}$ in corn is bound in the phytate complex, so its bioavailability in corn is very low. During dry-grind processing, particularly the fermentation step, some of the bonds that bind $\mathrm{P}$ to the phytate

\section{Table 4}

Ratios of streams vs. ground corn (fold of increase) in concentrations of different forms of phosphorus $(\mathrm{P})$ during dry-grind processing of corn into ethanol at three plants. $^{\text {A }}$

\begin{tabular}{|c|c|c|c|c|c|}
\hline Fraction name & Plant No. & Phytate P & Inorganic P & Rest of $\mathrm{P}$ & Total P \\
\hline \multirow{4}{*}{ Ground corn } & 1 & 1.00 & 1.00 & 1.00 & 1.00 \\
\hline & 2 & 1.00 & 1.00 & 1.00 & 1.00 \\
\hline & 3 & 1.00 & 1.00 & 1.00 & 1.00 \\
\hline & Mean & $1.00 \mathrm{f}$ & $1.00 \mathrm{e}$ & $1.00 \mathrm{c}$ & $1.00 \mathrm{e}$ \\
\hline \multirow[t]{4}{*}{ Cooked slurry } & 1 & 1.64 & 2.33 & 1.01 & 1.59 \\
\hline & 2 & 1.61 & 3.48 & 1.02 & 1.65 \\
\hline & 3 & 1.43 & 2.05 & 1.39 & 1.48 \\
\hline & Mean & $1.56 \mathrm{e}$ & $2.58 \mathrm{~d}$ & $1.13 \mathrm{c}$ & $1.57 \mathrm{~d}$ \\
\hline \multirow[t]{4}{*}{ Liquefied mash } & 1 & 1.41 & 2.56 & 0.76 & 1.40 \\
\hline & 2 & 1.64 & 3.05 & 1.01 & 1.63 \\
\hline & 3 & 1.44 & 2.06 & 1.15 & 1.45 \\
\hline & Mean & $1.49 \mathrm{e}$ & $2.53 \mathrm{~d}$ & $0.97 \mathrm{c}$ & $1.49 \mathrm{~d}$ \\
\hline \multirow[t]{4}{*}{ Fermented mash } & 1 & 2.33 & 11.39 & 5.39 & 3.66 \\
\hline & 2 & 2.73 & 11.73 & 7.22 & 4.36 \\
\hline & 3 & 2.58 & 11.03 & 7.16 & 4.14 \\
\hline & Mean & $2.54 \mathrm{c}$ & $11.37 \mathrm{c}$ & $6.59 \mathrm{a}$ & $4.04 \mathrm{~b}$ \\
\hline \multirow[t]{4}{*}{ Whole stillage } & 1 & 2.36 & 11.19 & 5.62 & 3.69 \\
\hline & 2 & 2.77 & 11.75 & 6.16 & 4.18 \\
\hline & 3 & 2.97 & 12.18 & 6.82 & 4.47 \\
\hline & Mean & $2.69 \mathrm{c}$ & $11.71 \mathrm{c}$ & $6.19 \mathrm{ab}$ & $4.11 \mathrm{~b}$ \\
\hline \multirow[t]{4}{*}{ Thin stillage } & 1 & 4.13 & 21.37 & 7.43 & 6.21 \\
\hline & 2 & 4.73 & 28.38 & 5.61 & 6.90 \\
\hline & 3 & 5.13 & 24.58 & 8.23 & 7.46 \\
\hline & Mean & $4.66 \mathrm{a}$ & $24.63 \mathrm{~b}$ & $7.05 \mathrm{a}$ & $6.85 \mathrm{a}$ \\
\hline \multirow[t]{4}{*}{ Distillers solubles } & 1 & 3.45 & 23.08 & 6.26 & 5.65 \\
\hline & 2 & 4.14 & 29.25 & 6.47 & 6.72 \\
\hline & 3 & 4.64 & 27.55 & 6.90 & 7.15 \\
\hline & Mean & $4.07 \mathrm{~b}$ & $26.53 \mathrm{a}$ & $6.53 \mathrm{a}$ & $6.49 \mathrm{a}$ \\
\hline \multirow{4}{*}{$\begin{array}{l}\text { Distillers wet } \\
\text { grains }\end{array}$} & 1 & 1.04 & 4.08 & 2.22 & 1.51 \\
\hline & 2 & 1.30 & 3.92 & 3.22 & 1.90 \\
\hline & 3 & 1.03 & 2.90 & 2.74 & 1.49 \\
\hline & Mean & $1.12 \mathrm{f}$ & $3.62 \mathrm{~d}$ & $2.74 \mathrm{bc}$ & $1.63 \mathrm{~d}$ \\
\hline \multirow[t]{4}{*}{ DDGS } & 1 & 1.69 & 11.55 & 3.39 & 2.85 \\
\hline & 2 & 1.91 & 11.00 & 3.74 & 3.03 \\
\hline & 3 & 1.81 & 9.81 & 5.63 & 3.20 \\
\hline & Mean & $1.80 \mathrm{~d}$ & $10.77 \mathrm{c}$ & $4.22 \mathrm{~b}$ & $3.02 \mathrm{c}$ \\
\hline
\end{tabular}

A Column means of three plants for each of nine fractions bearing different letters differ significantly at $p<0.05$. 
complex have been hydrolyzed. This would make more P available for absorption and result in greater P bioavailability in DDGS compared with corn.

\subsection{Differences among ethanol plants}

Among processing streams, all constituents examined exhibited significant differences in concentrations. However, differences in concentrations of minerals as well as different forms of P among ethanol plants varied with nutrients as well as processing streams. For ground corn, except for $\mathrm{Mg}$, Fe, phytate $\mathrm{P}$ and inorganic $\mathrm{P}$, all other nutrients had no significant difference among plants (Tables 1 and 3). However, for DDGS, more than half the attributes exhibited significant differences in concentrations among plants. This was also true for means of the nine fractions. Furthermore, for these constituents that had significant differences for both corn and DDGS, the pattern of differences for corn among the three plants did not match that for DDGS. These observations indicate that variation in DDGS mineral concentration and $P$ profile among plants resulted mainly from variation in processing conditions, and influence from corn composition was minimal.

Belyea et al. (2006) reported that the element content of corn did not differ among nine plants but that of DDGS did. Since plants used similar processing equipment to convert corn into ethanol and DDGS, they reasoned that variations in element contents of DDGS among plants must reflect differences in processing techniques and conditions. The present study mostly confirmed their findings.

\subsection{Comparison with published data for corn and DDGS}

As shown in Table 5, for corn, mineral concentrations in the present study were similar to Belyea et al. (2006). The report by Watson (1987) was based on several earlier studies in the 1950s and 1960s. We included here for reference only. Although the ranges were much larger in Watson (1987) than the later two studies, the average values were very similar for all minerals except for $\mathrm{Ca}, \mathrm{Na}$, and $\mathrm{Cu}$. For DDGS, concentrations of many minerals varied among studies, but data in the present study were closer to those of Spiehs et al. (2002) and Belyea et al. (2006) than Batal and Dale (2003). Variation in mineral concentrations among samples with studies, as expressed by coefficient of variation, is much larger in DDGS than in corn. Among the minerals in DDGS, all studies showed larger variation for $\mathrm{S}, \mathrm{Na}$ and $\mathrm{Ca}$, and $\mathrm{Fe}$. This comparison reaffirms the observation made earlier by Belyea et al. (2006) that the concentrations of most elements, including $\mathrm{P}$, at least in commercially grown corn have been conserved over the years, but a larger variation in DDGS mineral composition existed, reflecting influence by processing conditions.

As for P profile (Table 6), the present study showed that ground corn, on average, contained $2.12 \mathrm{mg} / \mathrm{g}$ phytate $\mathrm{P}, 0.26 \mathrm{mg} / \mathrm{g}$ inorganic $\mathrm{P}, 0.51 \mathrm{mg} / \mathrm{g}$ the rest of $\mathrm{P}$, and $2.89 \mathrm{mg} / \mathrm{g}$ total $\mathrm{P}$, dry matter

Table 5

Comparison of mineral concentrations in corn and DDGS among several reports.

\begin{tabular}{|c|c|c|c|c|c|c|c|c|c|c|c|}
\hline Source & $\begin{array}{l}\text { Statistical } \\
\text { Parameters }\end{array}$ & $\begin{array}{l}\mathrm{K} \\
(\mathrm{mg} / \mathrm{g})\end{array}$ & $\begin{array}{l}\mathrm{P} \\
(\mathrm{mg} / \mathrm{g})\end{array}$ & $\begin{array}{l}\mathrm{Mg} \\
(\mathrm{mg} / \mathrm{g})\end{array}$ & $\begin{array}{l}\mathrm{S} \\
(\mathrm{mg} / \mathrm{g})\end{array}$ & $\begin{array}{l}\mathrm{Na} \\
(\mathrm{mg} / \mathrm{g})\end{array}$ & $\begin{array}{l}\mathrm{Ca} \\
(\mathrm{mg} / \mathrm{g})\end{array}$ & $\begin{array}{l}\mathrm{Fe} \\
(\mu \mathrm{g} / \mathrm{g})\end{array}$ & $\begin{array}{l}\mathrm{Zn} \\
(\mu \mathrm{g} / \mathrm{g})\end{array}$ & $\begin{array}{l}\text { Mn } \\
(\mu \mathrm{g} / \mathrm{g})\end{array}$ & $\begin{array}{l}\mathrm{Cu} \\
(\mu \mathrm{g} / \mathrm{g})\end{array}$ \\
\hline \multicolumn{12}{|l|}{ Corn } \\
\hline \multirow{3}{*}{ Watson (1987) } & Minimum & 3.2 & 2.6 & 0.9 & 0.1 & 0.0 & 0.1 & 1.0 & 12.0 & 0.7 & 0.9 \\
\hline & Maximum & 7.2 & 7.5 & 1.4 & 2.2 & 1.5 & 1.0 & 100.0 & 30.0 & 54.0 & 10.0 \\
\hline & Mean & 3.7 & 2.9 & 1.2 & 1.2 & 0.3 & 0.3 & 30.0 & 14.0 & 5.0 & 4.0 \\
\hline \multirow[t]{5}{*}{ Belyea et al. (2006) } & Sample No. & 9 & 9 & 9 & 9 & 9 & 9 & 9 & 9 & 9 & 9 \\
\hline & Minimum & 3.51 & 2.64 & 1.07 & 1.06 & 0.00 & 0.05 & 21 & 18 & 4.7 & 1.3 \\
\hline & Maximum & 3.87 & 3.09 & 1.21 & 1.26 & 0.01 & 0.09 & 31 & 21 & 5.6 & 1.6 \\
\hline & Mean & 3.61 & 2.87 & 1.16 & 1.11 & 0.00 & 0.06 & 25.33 & 19.67 & 5.26 & 1.42 \\
\hline & $\mathrm{CV}(\%)^{\mathrm{a}}$ & 3.24 & 5.25 & 3.59 & 5.77 & 55.98 & 26.17 & 11.34 & 5.08 & 5.22 & 8.45 \\
\hline \multirow[t]{5}{*}{ The present study } & Sample No. & 3 & 3 & 3 & 3 & 3 & 3 & 10 & 3 & 3 & 3 \\
\hline & Minimum & 3.83 & 3.24 & 1.14 & 1.04 & 0.01 & 0.05 & 8.01 & 21.23 & 5.19 & 1.83 \\
\hline & Maximum & 4.10 & 3.54 & 1.31 & 1.08 & 0.01 & 0.09 & 10.25 & 23.42 & 5.36 & 2.01 \\
\hline & Mean & 3.92 & 3.39 & 1.21 & 1.07 & 0.01 & 0.07 & 9.02 & 22.34 & 5.32 & 1.92 \\
\hline & $\mathrm{CV}(\%)$ & 3.92 & 4.42 & 7.13 & 1.91 & 0.00 & 28.11 & 12.62 & 4.91 & 2.26 & 4.69 \\
\hline \multicolumn{12}{|l|}{ DDGS } \\
\hline \multirow[t]{5}{*}{ Spiehs et al. (2002) } & Sample No. & 118 & 118 & 118 & 118 & 118 & 118 & 118 & 118 & 118 & 118 \\
\hline & Minimum & 6.9 & 7.0 & 2.5 & 3.3 & 1.2 & 0.3 & 75.3 & 44.7 & 10.7 & 4.7 \\
\hline & Maximum & 10.6 & 9.9 & 3.7 & 7.4 & 5.1 & 1.3 & 156.4 & 312.0 & 21.3 & 7.6 \\
\hline & Mean & 9.4 & 8.9 & 3.3 & 4.7 & 2.4 & 0.6 & 119.8 & 97.5 & 15.8 & 5.9 \\
\hline & $\mathrm{CV}(\%)$ & 14.00 & 11.70 & 12.10 & 37.10 & 70.50 & 57.20 & 41.10 & 80.40 & 32.70 & 20.40 \\
\hline \multirow[t]{5}{*}{ Batal and Dale (2003) } & Sample No. & 12 & 12 & 12 & 12 & 12 & 12 & 12 & 12 & 12 & 12 \\
\hline & Minimum & 6.7 & 5 & 2.1 & 5.8 & 0.9 & 0.1 & 67 & 44 & 9 & 3 \\
\hline & Maximum & 9.9 & 7.7 & 3.3 & 11 & 4.4 & 7.1 & 325 & 88 & 48 & 18 \\
\hline & Mean & 9.1 & 6.8 & 2.8 & 8.4 & 2.5 & 2.9 & 149 & 61 & 22 & 10 \\
\hline & $\mathrm{CV}(\%)$ & 12.08 & 12.29 & 14.28 & 25.00 & 60.00 & 93.00 & 57.70 & 21.30 & 50.00 & 43.00 \\
\hline \multirow[t]{5}{*}{ Belyea et al. (2006) } & Sample No. & 9 & 9 & 9 & 9 & 9 & 9 & 9 & 9 & 9 & 9 \\
\hline & Minimum & 9.31 & 7.10 & 2.99 & 3.44 & 0.60 & 0.25 & 90.0 & 75.0 & 15.6 & 4.9 \\
\hline & Maximum & 12.40 & 9.43 & 3.79 & 8.27 & 2.30 & 0.34 & 109.0 & 170.0 & 19.3 & 6.8 \\
\hline & Mean & 11.22 & 8.52 & 3.48 & 5.76 & 1.30 & 0.28 & 98.7 & 113.7 & 17.0 & 5.6 \\
\hline & $\mathrm{CV}(\%)$ & 9.60 & 8.71 & 7.70 & 25.06 & 40.99 & 11.14 & 5.87 & 36.52 & 7.04 & 10.92 \\
\hline \multirow[t]{5}{*}{ The present study } & Sample No. & 3 & 3 & 3 & 4 & 3 & 3 & 3 & 3 & 3 & 3 \\
\hline & Minimum & 10.72 & 9.68 & 3.24 & 6.03 & 2.16 & 0.31 & 17.52 & 63.36 & 14.57 & 5.01 \\
\hline & Maximum & 12.42 & 10.18 & 3.63 & 7.94 & 2.94 & 0.48 & 26.63 & 67.28 & 17.98 & 6.07 \\
\hline & Mean & 11.44 & 9.98 & 3.45 & 6.83 & 2.63 & 0.37 & 21.47 & 65.15 & 15.81 & 5.55 \\
\hline & CV (\%) & 7.66 & 2.62 & 5.79 & 14.56 & 15.56 & 26.02 & 21.75 & 3.04 & 11.93 & 9.52 \\
\hline
\end{tabular}

${ }^{\text {a }} \mathrm{CV}=$ Coefficient of variation, also known as relative standard deviation. 
Table 6

Comparison of phosphorus $(\mathrm{P})$ profile in corn and DDGS among several reports.

\begin{tabular}{|c|c|c|c|c|c|c|c|}
\hline Source & $\begin{array}{l}\text { Statistical } \\
\text { Parameters }\end{array}$ & $\begin{array}{l}\text { Phytate }^{\mathrm{a}} \\
(\mathrm{mg} / \mathrm{g})\end{array}$ & $\begin{array}{l}\text { Phytate } \mathrm{P}^{\mathrm{a}} \\
(\mathrm{mg} / \mathrm{g})\end{array}$ & $\begin{array}{l}\text { Inorganic } \mathrm{P} \\
(\mathrm{mg} / \mathrm{g})\end{array}$ & $\begin{array}{l}\text { Rest of } \mathrm{P} \\
(\mathrm{mg} / \mathrm{g})\end{array}$ & $\begin{array}{l}\text { Total P } \\
(\mathrm{mg} / \mathrm{g})\end{array}$ & $\begin{array}{l}\text { Nonphytate } \mathrm{P}^{\mathrm{t}} \\
(\mathrm{mg} / \mathrm{g})\end{array}$ \\
\hline \multicolumn{8}{|l|}{ Corn } \\
\hline NRC (1994) & Mean & 7.1 & 2.0 & & & 2.8 & 0.8 \\
\hline \multirow[t]{5}{*}{ The present study } & Sample No. & 3 & 3 & 3 & 3 & 3 & 3 \\
\hline & Minimum & 7.06 & 1.99 & 0.23 & 0.49 & 2.75 & 0.76 \\
\hline & Maximum & 7.88 & 2.22 & 0.27 & 0.53 & 3.01 & 0.78 \\
\hline & Mean & 7.52 & 2.12 & 0.26 & 0.51 & 2.89 & 0.77 \\
\hline & $\mathrm{CV}(\%)^{\mathrm{c}}$ & 5.69 & 5.69 & 7.60 & 4.31 & 4.42 & 1.61 \\
\hline \multicolumn{8}{|l|}{$D D G S$} \\
\hline \multirow[t]{2}{*}{ Lumpkins and Batal (2005) } & Sample No. & 9 & 9 & & & 9 & 9 \\
\hline & Mean & 9.58 & 2.70 & & & 7.40 & 4.70 \\
\hline \multirow[t]{5}{*}{ Shetty et al. $(2008)^{\mathrm{d}}$} & Sample No. & 10 & 10 & & & 10 & 10 \\
\hline & Minimum & 13.10 & 3.69 & & & 7.39 & 2.20 \\
\hline & Maximum & 39.20 & 11.05 & & & 20.05 & 10.20 \\
\hline & Mean & 23.40 & 6.59 & & & 11.83 & 5.24 \\
\hline & $\mathrm{CV}(\%)$ & 31.28 & 31.28 & & & 35.97 & 49.84 \\
\hline \multirow[t]{5}{*}{ The present study } & Sample No. & 3 & 3 & 3 & 3 & 3 & 3 \\
\hline & Minimum & 13.34 & 3.76 & 2.56 & 1.76 & 8.35 & 4.56 \\
\hline & Maximum & 13.77 & 3.88 & 3.04 & 2.76 & 9.28 & 5.40 \\
\hline & Mean & 13.52 & 3.81 & 2.75 & 2.17 & 8.73 & 4.92 \\
\hline & $\mathrm{CV}(\%)$ & 1.67 & 1.67 & 9.34 & 24.07 & 5.60 & 8.79 \\
\hline
\end{tabular}

a To convert phytate $\mathrm{P}$ to phytate, use phytate $\mathrm{P} \times(660 /(31 \times 6))$; to convert phytate to phytate $\mathrm{P}$, use phytate $\times((31 \times 6) / 660)$.

b Nonphytate $\mathrm{P}=$ total $\mathrm{P}-$ phytate $\mathrm{P}$.

c $\mathrm{CV}=$ Coefficient of variation, also known as relative standard deviation.

d For nonphytate P, Shetty et al. (2008) used the term "free P". Total P for this study was calculated by adding phytate P and nonphytate P.

basis. The nonphytate $\mathrm{P}$ was calculated as $0.8 \mathrm{mg} / \mathrm{g}$. These values are similar to those of NRC (1994). In the present study, on average, DDGS contained $3.81 \mathrm{mg} / \mathrm{g}$ of phytate $\mathrm{P}, 2.75 \mathrm{mg} / \mathrm{g}$ of inorganic $P, 2.17 \mathrm{mg} / \mathrm{g}$ of the rest of $\mathrm{P}$, and $8.73 \mathrm{mg} / \mathrm{g}$ of total $\mathrm{P}$ (Table 3). The nonphytate $\mathrm{P}$ was calculated as $4.92 \mathrm{mg} / \mathrm{g}$. These values are closer to those of Lumpkins and Batal (2005) than Shetty et al. (2008). The latter had higher average values for all forms of P in DDGS. However, because the report by Shetty et al. (2008) also had much higher variation, the mean values for various forms of $P$ in the present study fall within the ranges of Shetty et al. (2008). Furthermore the concentration of total P in both corn and DDGS in the present study also agreed with Belyea et al. (2006) but for the rest of $\mathrm{P}$ in corn or DDGS, no previous reports are available for comparison.

\section{Conclusions}

This study was designed to identify specific steps in the drygrind ethanol process, which cause significant changes in mineral composition and phosphorus profile. Compared with ground corn, all minerals increased in concentrations in downstream products, but the extent of increase varied greatly with individual streams and minerals. Fermentation caused most dramatic changes due to starch depletion, but other steps, such as slurring/cooking, centrifuging, and evaporation, also led to significant changes. However, changes brought by these other steps were mostly physical in nature. Among downstream products, thin stillage had the highest concentrations of all minerals while distillers wet grains had the lowest.

\section{References}

Amezcua, C.M., Parsons, C.M., Noll, S.L., 2004. Content and relative bioavailability of phosphorus in distillers dried grains with solubles in chicks. Poultry Sci. 83, 971-976.

AOAC (Association of Official Analytical Chemists), 2002. AOAC Official Methods of Analysis. AOAC International, Arlington, VA.
Batal, A., Dale, N., 2003. Mineral composition of distillers dried grains with solubles. J. Appl. Poultry Res. 12, 400-403.

Belyea, R.L., Clevenger, T.E., Singh, V., Tumbleson, M.E., Rausch, K.D., 2006. Element concentrations of dry-grind corn-processing streams. Appl. Biochem. Biotechnol. 134, 113-128.

Bohn, L., Meyer, A.S., Rasmussen, S.K., 2008. Phytate: impact on environment and human nutrition. A challenge for molecular breeding. J. Zhejiang Univ. Sci. B 9 (3), 165-191.

Bothast, R.J., Schlicher, M.A., 2005. Biotechnological processes for conversion of corn into ethanol. Appl. Microbiol. Biotechnol. 67, 19-25.

Chen, P.S., Toribara, T.Y., Warner, H., 1956. Microdeterminations of phosphorus. Anal. Chem. 28, 1756-1758.

Gould, D.H., 1998. Polioencephalomalacia. J. Anim. Sci. 76, 309-314.

Han, J.C., Liu, K.S., 2010. Changes in proximate composition and amino acid profile during dry grind ethanol processing from corn and estimation of yeast contribution toward DDGS proteins. J. Agric. Food Chem. 58, 3430-3437.

He, Z.Q., Waldrip, H.W., Honeycutt, C.W., Erich, M.S., Senwo, Z.N., 2009. Enzymatic quantification of phytate in animal manure. Commun. Soil Sci. Plant Anal. 40, 566-575.

Koelsch, R., Lesoing, G., 1999. Nutrient balance on Nebraska livestock confinement systems. J. Anim. Sci. 77, 63-71.

Liu, K.S., Peterson, K.L., Raboy, V., 2007. Comparison of the phosphorus and mineral concentrations in bran and abraded kernel fractions of a normal barley (Hordeum vulgare) cultivar versus four low phytic acid isolines. J. Agric. Food Chem. 55, 4453-4460.

Lott, J.N.A., Ockenden, I., Raboy, V., Batten, G.D., 2000. Phytic acid and phosphorus in crop seeds and fruits: global estimate. Seed Sci. Res. 10, 11-33.

Lumpkins, B.S., Batal, A.B., 2005. The bioavailability of lysine and phosphorus in distillers dried grains with solubles. Poultry Sci. 84, 581-586.

Moreau, R.A., Liu, K.S., Winkler-Moser, J.K., Singh, V., 2010. Changes in lipid composition during dry grind ethanol processing of corn. J. Am. Oil. Chem. Soc. doi:10.1007/s11746.010.1674.y.

Niles, G.A., Morgan, S., Edwards, W.C., Lalman, D., 2002. Effects of dietary sulfur concentrations on the incidence and pathology of polioencephalomalacia in weaned beef calves. Vet. Hum. Toxicol. 44, 70-72.

Noureddini, H., Malik, M., Byun, J., Ankeny, A.J., 2009. Distribution of phosphorus compounds in corn processing. Bioresour. Technol. 100, 731-736.

NRC (National Research Council), 1994. Nutrient Requirements of Poultry, ninth ed. National Academy Press, Washington, DC.

NRC (National Research Council), 1998. Nutrient Requirements of Swine, 10th ed. National Academy Press, Washington, DC. pp. 110-123.

Pedersen, C., Boersma, M.G., Stein, H.H., 2007. Digestibility of energy and phosphorus in ten samples of distillers dried grains with solubles fed to growing pigs. J. Anim. Sci. 85, 1168-1176.

Rausch, K.D., Raskin, L.M., Belyea, R.L., Agbisit, R.M., Daugherty, B.J., Clevenger, T.E., Tumbleson, M.E., 2005. Phosphorus concentrations and flow in maize wet milling streams. Cereal Chem. 82, 431-435. 
Shetty, J.K., Paulson, B., Pepsin, M., Chotani, G., Dean, B., Hruby, M., 2008. Phytate in fuel ethanol production offers economical and environmental benefits. Int. Sugar J. 110, 160-174

Spiehs, M.J., Whitney, M.H., Shurson, G.C., 2002. Nutrient database for distiller's dried grains with solubles produced from new ethanol plants in Minnesota and South Dakota. J. Anim. Sci. 80, 2639-2645.
Spiehs, M.J., Varel, V.H., 2009. Nutrient excretion and odorant production in manure from cattle fed corn wet distillers grains with solubles. J. Anim. Sci. 87, 29772984

Watson, S.A., 1987. Structure and composition. In: Watson, S.A., Ramstad, P.E. (Eds.) Corn: Chemistry and Technology. AACC, Inc., St. Paul, MN, pp. 53-82 (Chapter 3). Wodzinski, R.J., Ullah, A.H., 1996. Phytase. Adv. Appl. Microbiol. 42, 264-303. 